\title{
SYNTHESIS AND CHARACTERIZATION OF DERIVATIZED CARBON NANOSTRUCTURES
}

\author{
TIMEA-ANITA DULL-SZABÓa, MELINDA-EMESE FÜSTÖS ${ }^{a}$, \\ MARIA SUCIU ${ }^{\mathrm{b}}$, GABRIEL KATONA ${ }^{\mathrm{a}, *}$
}

\begin{abstract}
Carbon nanotube-triethanolamine and carbon nanotubedendrimer structures were obtained from carboxyl functionalized nanotubes (SW-, DW-, MW-COOH). The synthesized compounds were characterized by elemental analysis, scanning transmission electron microscopy coupled with energy-dispersive X-ray spectroscopy (STEM-EDX). The toxicity of the obtained derivatized carbon nanostructures was tested on human immortalized keratinocyte cell line (HaCaT). The viability of the cells was determined using the 3-[4,5-dimethylthiazol-2-yl]-2,5-diphenyl-tetrazolium-bromide (MTT) cell proliferation assay.
\end{abstract}

Keywords: carbon nanotube, dendrimer, biological activity, MTT assay, $\mathrm{HaCaT}$

\section{INTRODUCTION}

Carbon nanotubes (CNTs) are hollow cylinders derived from rolled sheets of graphene. These nanostructures belong to the fullerene family, the third allotropic form of carbon; have unique electrical, mechanical and optical properties [1].

Three types of carbon nanotubes are distinguished based on the number of graphene sheets rolled upon itself: single-walled carbon nanotubes (SWCNTs), double-walled (DWCNTs) and multi-walled CNTs (MWCNTs). Different methods are used for the derivatization of nanotubes: covalent and non-covalent functionalization. Due to functionalization, problems such as

\footnotetext{
a Babeş-Bolyai University, Faculty of Chemistry and Chemical Engineering, Arany János street no 11, 400028 Cluj-Napoca, Romania

b Babeş-Bolyai University, Faculty of Biology and Geology, Gh. Bilașcu street no 44, ClujNapoca, Romania

*Corresponding author: gabik@chem.ubbcluj.ro
} 
agglomeration and bundle formation can be avoided, which enhances their dispersion in organic solvents. A good dispersion of nanotubes can be also induced by the use of surfactants [2].

Carbon nanotubes found application in many fields, being suitable for biomedical application thanks to their low toxicity. Different type of nanotubes can be used in drug delivery, gene delivery or anticancer therapy [3].

Dendrimers are hyperbranched, monodisperse macromolecules with nanometer-scale dimension [4]. The solubility and reactivity of dendrimers depend on the nature and number of functional groups on their periphery. Biomedical application is the most important utilization of dendrimers. They can be used as contrast agents in magnetic resonance imaging (MRI), as carriers in drug delivery and gene therapy and also as catalysts in industrial processes [5].

Combination of carbon nanotubes and dendrimers via covalent linkage leads to structures with unique properties and low toxicity. Poliamidoamine dendrimers (PAMAM) covalently attached to the surface of MWCNTs were described by B. Zhanga et al. [6]. According to their results, the MWCNT-PAMAM hybrids showed good dispersibility and activity in aqueous solution. Synthesis of PAMAM G4.0 dendrimer-functionalized carbon nanotubes was reported by Yang et al. [7]. They investigated the cellular toxicity of the obtained structures on HeLa cells and found that MWCNTPAMAM-G1 is the least toxic and the functionalized nanotubes can be used as vectors to deliver pEGFP-N1 into HeLa cells.

Herein we present the cytotoxicity investigation of some derivatized carbon nanostructures obtained upon covalent functionalization of different types of carbon nanotubes with the commercially available triethanolamine and also with the zero-generation dendrimer synthesized according to the procedure described previously [8]. The cytotoxicity of these species was studied on HaCaT cell line using the MTT cell proliferation assay. The derivatized nanostructures can be used in catalysis or in medicine as supports for drug delivery.

\section{RESULTS AND DISCUSSION}

The synthesized structures were characterized by elemental analysis and STEM-EDX technique, respectively.

It is noticeable, that the $\mathrm{N}$ content of the functionalized carbon nanotubes is significantly higher compared to the nitrogen content of the substrates (SW-, DW-, MW-COOH) which originates from the air while sample preparation, confirming the covalent linkage of the dialkylamino-linker molecules and the $\mathrm{N}$-atom containing compounds (triethanolamine, dendrimer). 
The functionalized double-wall carbon nanotubes were further characterized by STEM-EDX technique. This technique allows the determination of the elemental composition at a well-defined position of the transmission electron microscopy pictogram. In Figure 1 is presented the TEM-EDX analysis of the DW-COOH. The spectra indicate the presence of carboxyl groups on the surface of the nanotubes, but no nitrogen content is detected. The copper signs originate from the sample holder (copper grid) in each case.
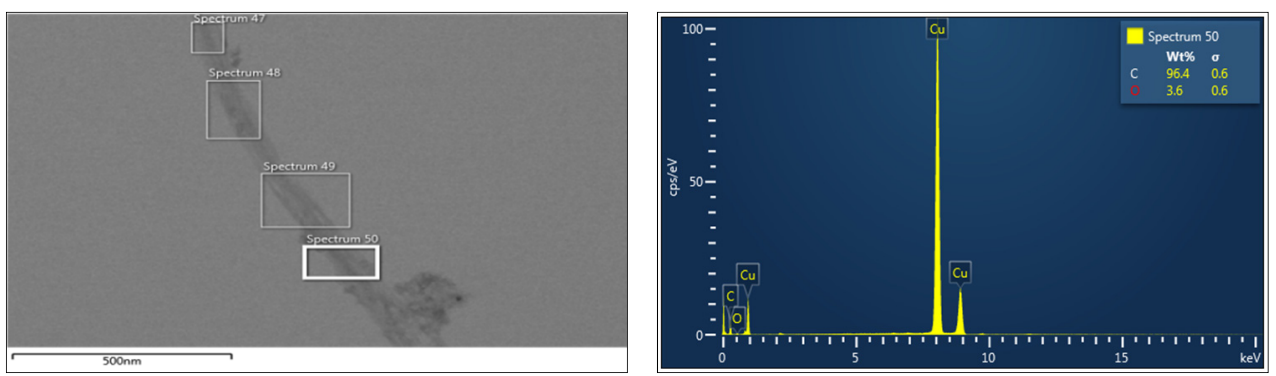

Figure 1. TEM-EDX spectra of DW-COOH

In Figure 2 is depicted the TEM image and the corresponding EDX spectra of the dendrimer functionalized double-wall carbon nanotubes (DW$\mathrm{TMB}$ ). The presence of $\mathrm{N}$ atom in the structure proves the covalent attachment of the dendrimer to the surface of the nanotubes.
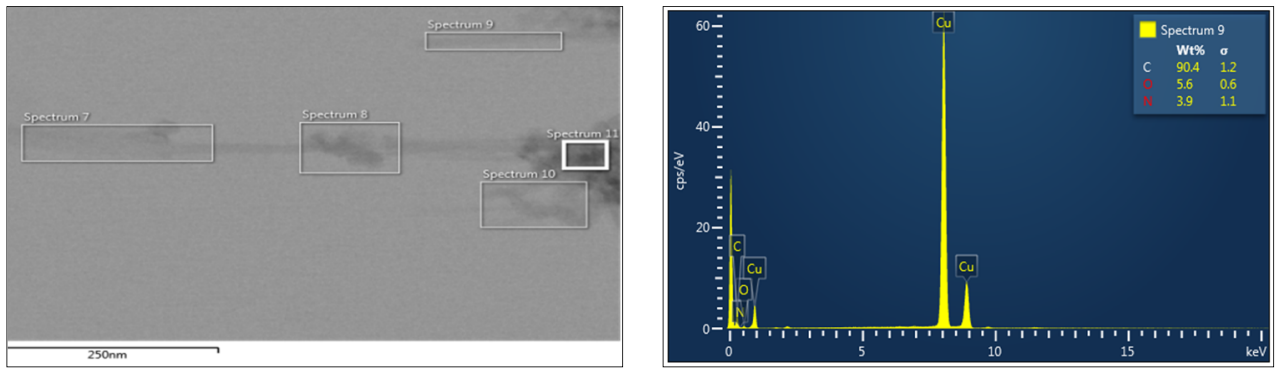

Figure 2. TEM-EDX spectra of DW-TMB 
The TEM-EDX spectra of DW-TEA structure is presented in Figure 3. The EDX analysis results prove the functionalization of the carbon nanotubes with triethanolamine by the detection of the $\mathrm{N}$ atom.
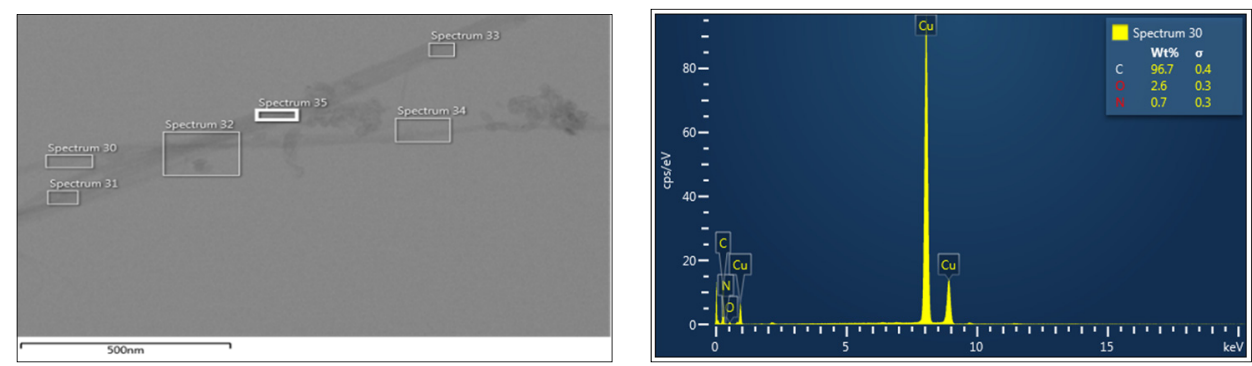

Figure 3. TEM-EDX spectra of DW-TEA

The toxicity of the obtained derivatized carbon nanostructures was investigated on HaCaT cell line. In Figure $\mathbf{4}$ and Figure $\mathbf{5}$ is represented the viability of the cells in the presence of different derivatized nanostructures. In each graphic CA symbolizes the so-called positive control (the cells are in their media without surfactant or carbon nanostructures) and 0 stands for the negative control (a $0.05 \%$ solution of Triton X-100 added to the cell media without carbon nanostructures).

In case of the two nanotube-linker species (Figure 4), the DAOfunctionalized nanotubes (5d-f) presented lower toxicity on the studied cell line than the CNT-DAPr.

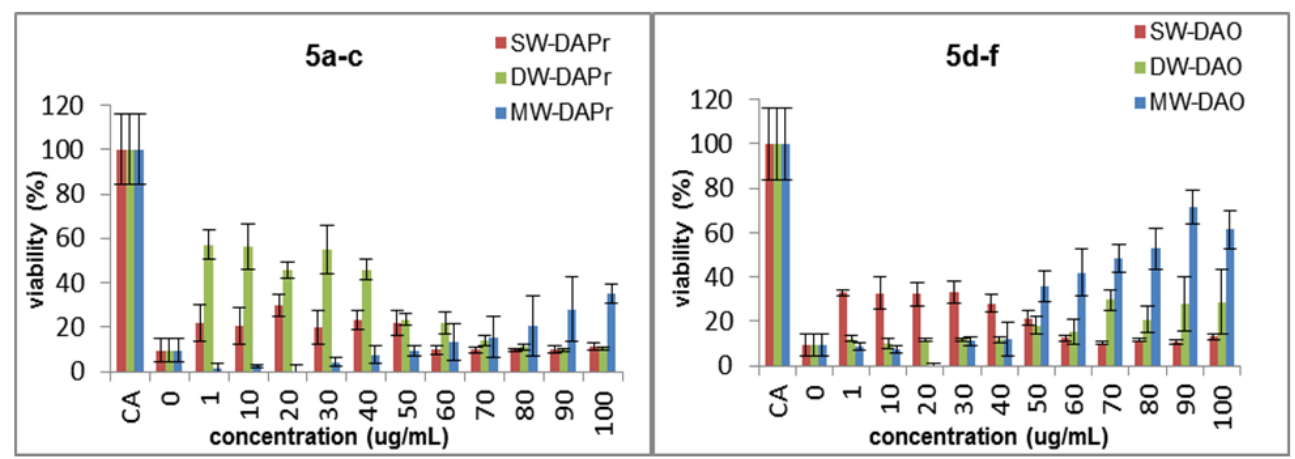

Figure 4. Cytotoxicity of 5a-c and $5 \mathrm{~d}-\mathrm{f}$ nanostructures 
The TEA functionalized nanotubes $(\mathbf{8 d}-\mathbf{f})$ seemed to be the most toxic nanostructures. In the case of dendrimer modified nanotubes the double-wall carbon nanotube species (11e) presented the lowest cytotoxicity (Figure 5).

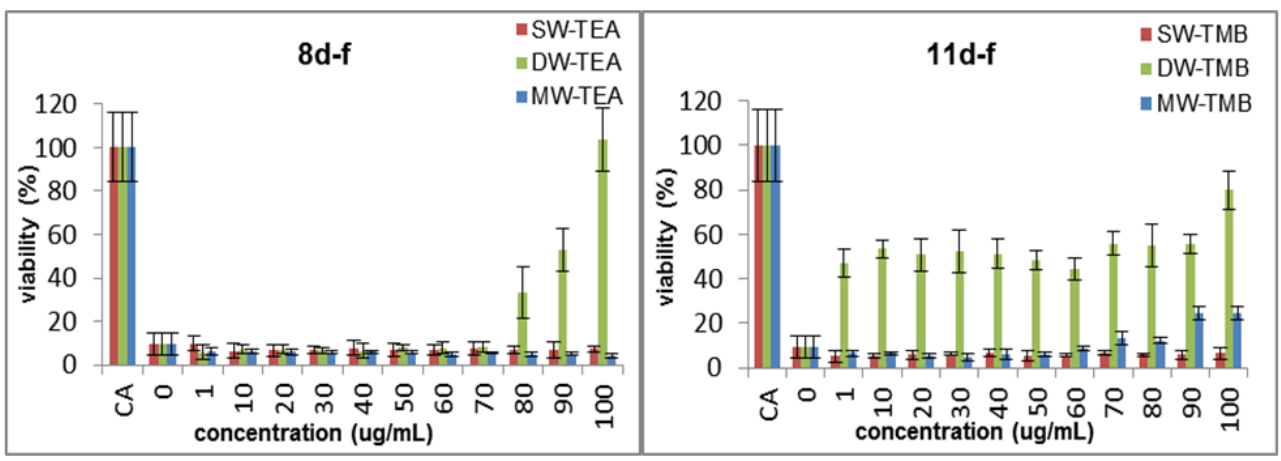

Figure 5. Cytotoxicity of $8 d-f$ and $11 d-f$ nanostructures

\section{CONCLUSIONS}

The covalent functionalization of the carbon nanotubes with $\mathrm{N}$ atom containing compounds: 1,3-diaminopropane, 1,8-diaminooctane, triethanolamine and the zero generation dendrimer, was confirmed by elemental and TEM-EDX analysis.

The cytotoxicity test results on the investigated $\mathrm{HaCaT}$ cell line indicated that in the presence of the surfactant the cell viability decreased up to $20 \%$. By adding the derivatized carbon nanostructures in different concentrations this toxicity was overcome and cell viability increased almost in each case. The best results were obtained in case of the dendrimer functionalized double walled carbon nanotubes added to the cell media in high concentration.

\section{EXPERIMENTAL SECTION}

In order to synthesize the desired nanotube-dendrimer and the nanotube-triethanolamine structures, respectively, in the first step two diaminoalkyl linker-like molecules were attached covalently to the carbon nanotubes scaffold using N,N'-carbonyldiimidazole (CDI) as an activating agent for the carboxyl functional groups. Scheme 1 illustrates the synthesis steps for obtaining the CNT-DAPr and CNT-DAO species. 


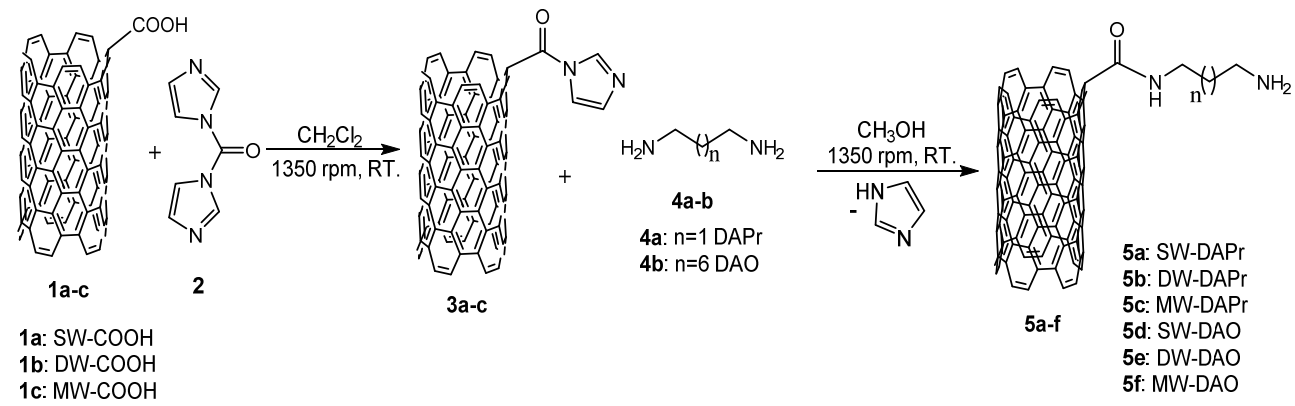

Scheme 1. Functionalization of CNTs with diaminoalkyl-linkers

\section{Synthesis of nanotube-linker (5a-f) structures}

In a $50 \mathrm{~mL}$ Falcon tube $200 \mathrm{mg}$ carboxyl derivatized carbon nanotubes (single-, double- and multi-walled) were suspended in $30 \mathrm{~mL}$ dry dichloromethane and sonicated for 15 minutes. $500 \mathrm{mg} \mathrm{CDI}$ dissolved in dry dichloromethane $(5 \mathrm{~mL})$ was added to the suspension. The mixture was left overnight on shaker at 1350 rpm with occasional sonication to avoid bundling.

The 3a-c nanotube derivatives were filtered on PTFE membrane (pore size $0.22 \mu \mathrm{m}$ ) and washed with dichloromethane repeatedly until the complete removal of the unreacted $\mathrm{CDI}$ and the produced imidazole. The procedure was monitored by thin layer chromatography (TLC) using dichloromethane: methanol 9:1 eluent mixture $\left(R_{f}=0.54\right)$. The product was dried at room temperature and used further being suspended in $10 \mathrm{~mL}$ methanol and sonicated for 20 minutes. $200 \mathrm{mg}$ diaminoalkyl derivative (DAPr, DAO) was dissolved in methanol and added to the suspension. The mixture was left overnight on shaker at 1350 rpm at room temperature.

The derivatized nanotubes were separated from the suspension by vacuum filtration, washed with methanol repeatedly in order to remove the unreacted diaminoalkyl derivatives and dried in vacuo.

\section{Synthesis of carbon nanotube-triethanolamine (8d-f) structures}

The synthesis steps are depicted in Scheme 2. Triethanolamine was covalently bonded to the linker-type carbon nanotube species while imidazole was formed as by-product. 


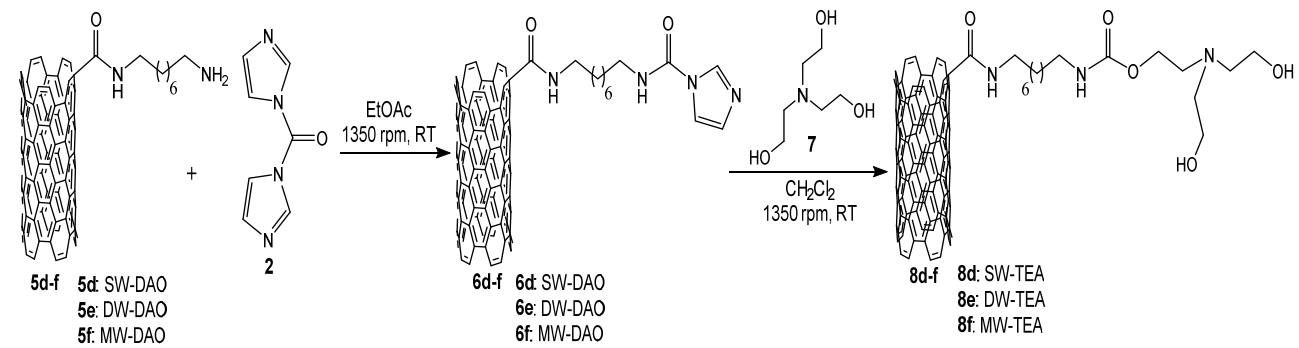

Scheme 2. Synthesis of the $8 \mathrm{~d}-\mathrm{f}$ derivatized structures

The 8d-f conjugates were synthesized according to the following procedure. $50 \mathrm{mg}$ diaminooctane-functionalized carbon nanotubes (SW-DAO, DW-DAO, MW-DAO) were suspended in $10 \mathrm{~mL}$ ethyl acetate and sonicated for 20 minutes. $100 \mathrm{mg}$ CDI dissolved in ethyl acetate was added to the suspension. The mixture was left overnight on shaker at $1350 \mathrm{rpm}$ with ocasional sonication. The $\mathbf{6 d - f}$ compounds were separated from the suspension by vacuum filtration and washed with ethyl acetate repeatedly until no CDI was detectable in the filtrate on the TLC plate.

Further the dried carbon nanotubes were suspended in $10 \mathrm{~mL}$ dichloromethane and $150 \mathrm{mg}$ TEA was added. The suspension was left overnight on shaker (1350 rpm). The functionalized carbon nanotubes were filtered off and washed with dichloromethane to eliminate the excess of the triethanolamine. The removal of the triethanolamine adsorbed on the surface of the carbon nanotubes was monitorized by $\mathrm{TLC}$ in $\mathrm{CH}_{2} \mathrm{Cl}_{2}: \mathrm{CH}_{3} \mathrm{OH} 8: 2$ eluent mixture $\left(\mathrm{R}_{\mathrm{f}}=\right.$ $0.48)$.

\section{Synthesis of CNTs-TMB-DEA OG (11d-f) conjugates}

In Scheme 3 is illustrated the functionalization of CNTs-DAO species with an aromatic core $\mathrm{G} 0$ dendrimer (TMB).

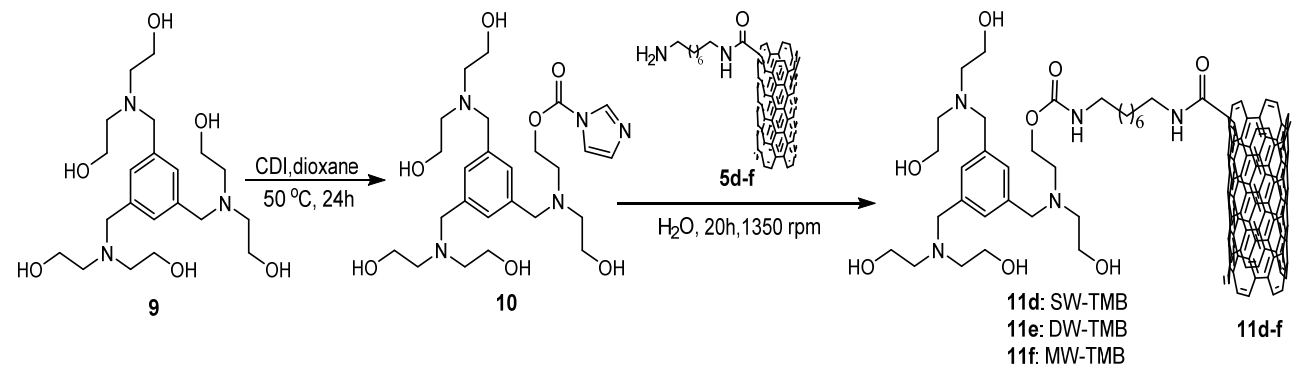

Scheme 3. Synthesis of the CNTs-dendrimer nanostructures 
$318 \mathrm{mg}$ dendrimer was dissolved in $30 \mathrm{~mL}$ dry dioxane. The mixture was heated at $50{ }^{\circ} \mathrm{C}$ and $100 \mathrm{mg} \mathrm{CDI}$ was added. The reaction mixture was stirred for 24 hours at $50{ }^{\circ} \mathrm{C}$. The solvent was evaporated on rotary evaporator and the remained viscous substance was used in the next step without further purification. Compound $\mathbf{1 0}$ dissolved in water was added to an aqueous suspension of $100 \mathrm{mg}$ diaminooctane functionalized nanotubes. After sonication for 30 minutes, the suspension was left on shaker at $1350 \mathrm{rpm}$ overnight. The functionalized nanotubes $11 \mathrm{~d}$-f were removed from the suspension by vacuum filtration and washed with water and acetone, respectively. The obtained nanotube-dendrimer species were dried at room temperature.

In Table 1 are presented the results of the elemental analysis.

Table 1. Results of the elemental analysis

\begin{tabular}{|c|c|c|c|}
\hline Sample & $\mathbf{N} \%$ & $\mathbf{C} \%$ & $\mathbf{H} \%$ \\
\hline SW-COOH & 0,23 & 91,72 & 1,20 \\
\hline SW-DAPr & 1,03 & 90,31 & 1,21 \\
\hline SW-DAO & 1,04 & 92,94 & 1,54 \\
\hline DW-COOH & 0,18 & 89,29 & 0,96 \\
\hline DW-DAPr & 1,24 & 91,73 & 1,33 \\
\hline DW-DAO & 0,99 & 94,01 & 1,49 \\
\hline DW-TEA & 1,23 & 89,39 & 1,98 \\
\hline MW-COOH & 0,19 & 86,49 & 1,25 \\
\hline MW-DAPr & 1,11 & 90,45 & 1,38 \\
\hline MW-DAO & 1,12 & 90,50 & 1,57 \\
\hline MW-TMB & 1,36 & 89,50 & 1,89 \\
\hline \multicolumn{4}{|r}{}
\end{tabular}

\section{Sample preparation for biological testing}

$10 \mathrm{mg}$ derivatized carbon nanostructure (5a-f, 8d-f, 11d-f) was suspended in $10 \mathrm{~mL} 0.05 \%$ Triton $\mathrm{X}-100$ aqueous solution. Each sample was sonicated for 30 minutes and stored in the fridge until testing.

The HaCaT cells were cultivated in a freshly made media consisting of $88 \%$ DMEM (Dulbecco's Modified Eagle Medium), 10\% fetal calf serum (FCS), 1\% penicillin/streptomycin and 1\% L-glutamine.

\section{Determination of cell viability}

The viability of the cells was determined using the colorimetric MTT cell proliferation assay. The cells were incubated in plates of 96 wells for 24 hours and the prepared nanostructures were added in different concentrations 
(range 1-100 ug/mL). After the repeated incubation, MTT solution was added to each well (10 uL, $5 \mathrm{mg} / \mathrm{mL}$ in phosphate buffer) and the samples were incubated at $37^{\circ} \mathrm{C}$ for 90 minutes (humidity $\sim 90 \%, \mathrm{CO}_{2}$ content $\sim 5 \%$ ). The formed insoluble purple formazan crystals were dissolved by adding an acidified isopropanol solution $(40 \mathrm{mM} \mathrm{HCl}, 0.1 \%$ Triton X-100). The absorbance of samples was measured at $550 \mathrm{~nm}$. The reference wavelength used was at $630 \mathrm{~nm}$. Each sample absorbance was measured consecutive 5 times and the average value was used for the determination of the number of viable cells, which can be expressed with the following formula:

$$
\text { Viability }(\%)=\left(A_{S} * 100\right) / A_{C A} \text {, }
$$

where $\mathbf{A}_{\mathbf{S}}$ is the absorbance of the sample; $\mathbf{A}_{\mathbf{C A}}$ is the absorbance of the positive control.

\section{Instruments used}

The thin layer chromatography analysis was accomplished on Silica Merck TLC $60 \mathrm{~F}_{254}$ aluminum plates. The elemental analysis measurements were performed on vario MICRO cube $\mathrm{CHN}$ elemental analyzer. The transmission electron microscopy images were recorded on STEM HD 2700 Hitachi microscope with EDX system. The cytotoxicity was measured with a BioTek Synergy HT plate reader using Gen5 Plate Reader software.

\section{ACKNOWLEDGMENTS}

This research was possible with the financial support of the PN-II-ID-PCE2011-3-0346 project.

\section{REFERENCES}

1. S. Vardharajula, Sk. Z. Ali, P. M. Tiwari, E. Eroğlu, K. Vig, V.A. Dennis, S.R. Singh, Int J Nanomedicine, 2012, 7, 5361-5374.

2. K. S. Ibrahim, Carbon Letters, 2013, 14(3), 131-144.

3. H. Sadegh, R. Shahryari-ghoshekandi, Nanomed. J., 2015, 2(4), 231-248.

4. U. Gupta, O. Perumal, "Dendrimers and Its Biomedical Applications", Natural

5. and Synthetic Biomedical Polymers, 2014, chapter 15.

6. B. Klajnert, M. Bryszewska, Acta Biochimica Polonica, 2001, 48, 199-208. 
TIMEA-ANITA DULL-SZABÓ, MELINDA-EMESE FÜSTÖS, MARIA SUCIU, GABRIEL KATONA

7. B. Zhanga, Q. Chena, H. Tanga, Q. Xiea, M. Maa, L. Tana, Y. Zhanga, S. Yaoa, Colloids and Surfaces B: Biointerfaces, 2010, 80, 18-25.

8. K. Yang, W. Qin, H. Tang, L. Tan, Q. Xie, M. Ma, Y. Zhang, S. Yao, J Biomed Mater Res Part A, 2011, 99A, 231-239.

9. M. E. Füstös, T. A. Sipos, M. V. Diudea, G. Katona, Croat. Chem. Acta, 2015, 88 (2), 129-132. 